\title{
Advanced Scanning Probe Nanolithography Using GaN Nanowires
}

Mahmoud Behzadirad ${ }^{\dagger *}$, Stephan Mecholdt ${ }^{\dagger}$, , John N. Randall ${ }^{\square}$, Joshua B. Ballard ${ }^{\square}$, James Owen ${ }^{\square}$, Ashwin K. Rishinaramangalam ${ }^{\dagger}$, Alexander Reum ${ }^{\ddagger}$, Teodor Gotszalk ${ }^{\square}$, Daniel F. Feezell $^{\dagger}$, Ivo W. Rangelow ${ }^{\dagger}$, , Tito Busani ${ }^{\dagger *}$.

$\dagger$ Center for High Technology Materials (CHTM), University of New Mexico (UNM), Albuquerque, New Mexico 87106, United States.

${ }^{\dagger}$ Group of Nanoscale Systems, Technische Universität Ilmenau, Gustav-Kirchhoff-Straße1, 98693 Ilmenau, Germany.

$\square$ Zyvex Labs., 1301 N Plano Rd, Richardson, TX 75081, United States.

$\$$ Nano analytik GmbH, Ehrenbergstr. 1, 98693 Ilmenau, Germany.

Department of Nanometrology, Faculty of Microsystem Electronics and Photonics, Wroclaw University of Science and Technology, Janiszewskiego 11/17 Street, 50-372 Wroclaw, Poland. 


\section{Sample preparation and synthesis process of GaN NWs}

To grow GaN NWs through a bottom-up approach, a GaN template on sapphire were coated by a thin silicon nitride $\left(\mathrm{Si}_{3} \mathrm{~N}_{4}\right)$ film. The thickness of the dielectric film can vary from 50 to $100 \mathrm{~nm}$. Then a thin layer of antireflection coating (i-Con-7 or i-Con-16) followed by NR7-500 resist were spin coated on the sample to prepare it for interferometric lithography (IL). Using IL setup (355 $\mathrm{nm}$ laser), circular holes with diameter of $200 \mathrm{~nm}$ were pattern on the photoresist. Then $\mathrm{O}_{2}$-plasma was applied to etch underneath antireflection coating layer followed by ICP plasma etching (using $\mathrm{CF}_{4}$ gas) to transfer photoresist pattern to the dielectric film. Afterward, sample was cleaned in Piranha $\left(\mathrm{H}_{2} \mathrm{O}_{2}: \mathrm{H}_{2} \mathrm{SO}_{4}, 1: 4\right)$ solution three times each for $10 \mathrm{~min}$ to lift-off photoresist and antireflection coating layers, followed by 1 min cleaning in $\mathrm{H}_{2} \mathrm{O}: \mathrm{HCL}(3: 1)$ solution to remove native oxide. Then, sample was load to MOCVD reactor to grow GaN NWs in opening areas. Figure S1 schematically demonstrate the procedure needed to follow to grow GaN NWs used in this research.

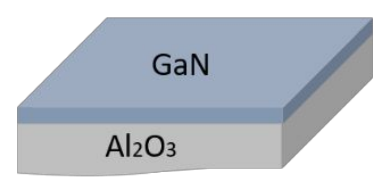

I. GaN on Sapphire template

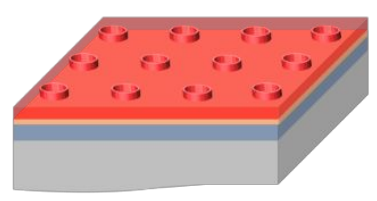

IV. IL lithography

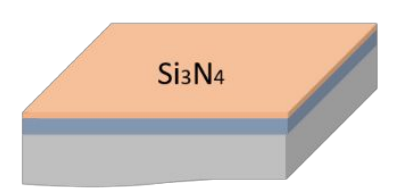

II. Dielectric deposition

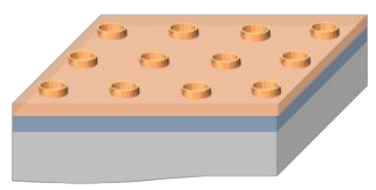

V. Transferring pattern into dielectric

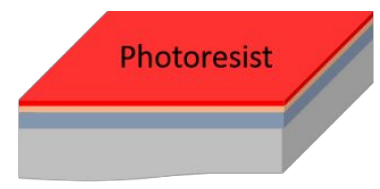

III. Photoresist spin coating

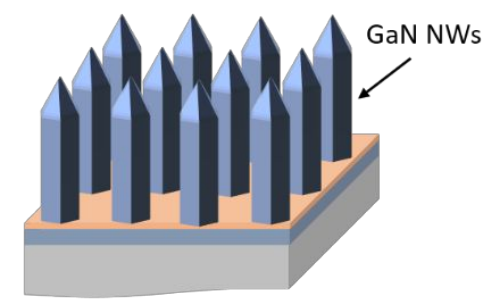

VI. Epitaxial growth of GaN NWs

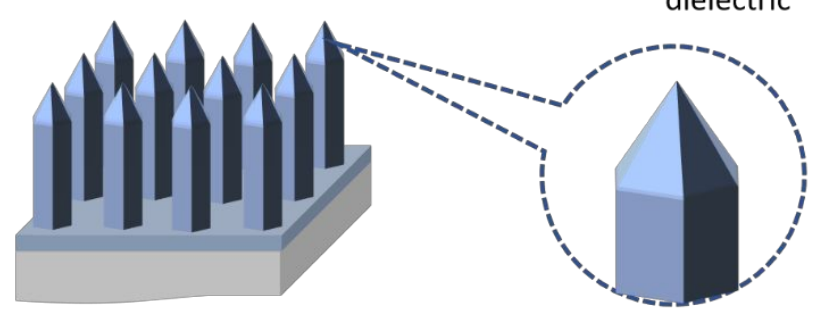

VII. Removing dielectric

Figure S1. Fabrication process of selective area GaN NWs through a bottom-up approach. 


\section{TEM images to compare radius of curvature and cone angles of the GaN NW and $W$ tips}

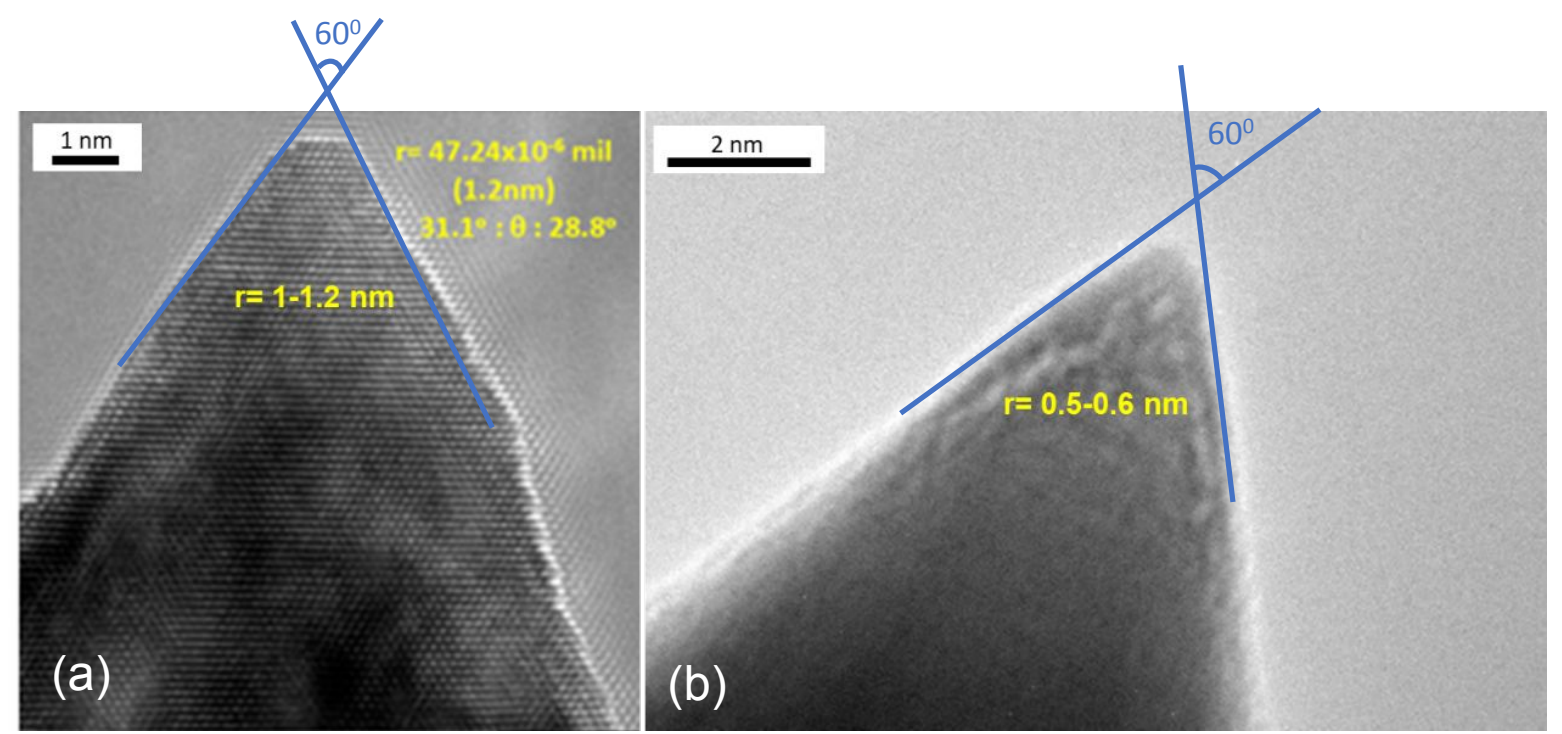

Figure S2. TEM images of (a) a standard W-tip, and (b) a GaN tip.

\section{AFM and STM probe fabrication in focus ion beam (FIB)/scanning electron microscopy (SEM)}

Following figures demonstrate steps to fabricated GaN STM and AFM probes using FIB/SEM. The process starts with flattening the $\mathrm{W}$ - and Si-tip. Then by means of an omniprobe, a GaN NW is detached from the as-grown substrate and transferred to the flattened tips, as seen in Figure S3 and S4 (b)-(c). The process will be completed by Pt deposition to weld NWs to the STM and AFM probes flattened substrate.
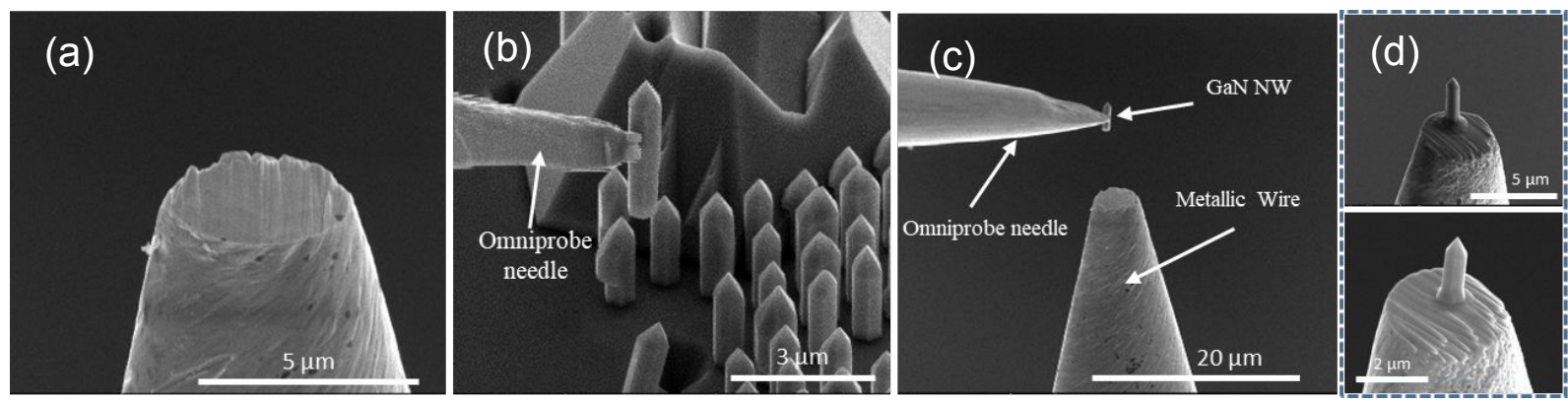

Figure S3. (a) Flattened W-tip by FIB. (b) Grabbing GaN NW using omni-probe. (c) transferring and positioning NW on the flattened W-tip. (d) Two examples of fabricated GaN STM probes. 

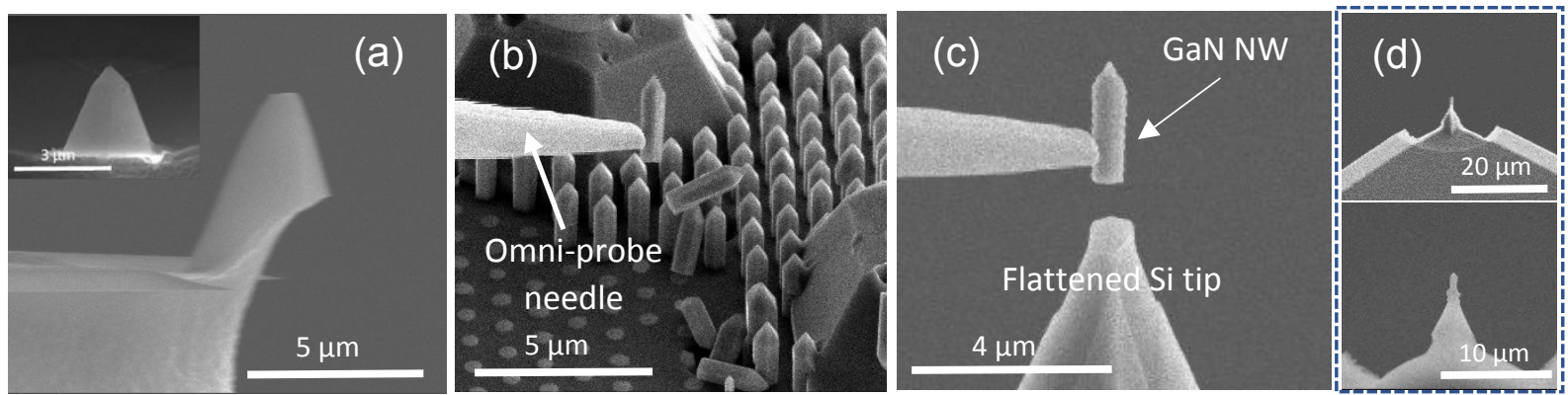

Figure S4. (a) Flattened Si-tip on active cantilever by FIB. (b) Grabbing GaN NW using omni-probe. (c) transferring and positioning NW on the flattened Si-tip. (d) Two examples of fabricated GaN AFM probes.

\section{Active Si Cantilevers}

An active cantilever contains of piezoelectric read-out and thermomechanical actuator to assist AFM for acquiring higher resolution images ${ }^{1-3}$. The Cantilever oscillates at its resonant frequency

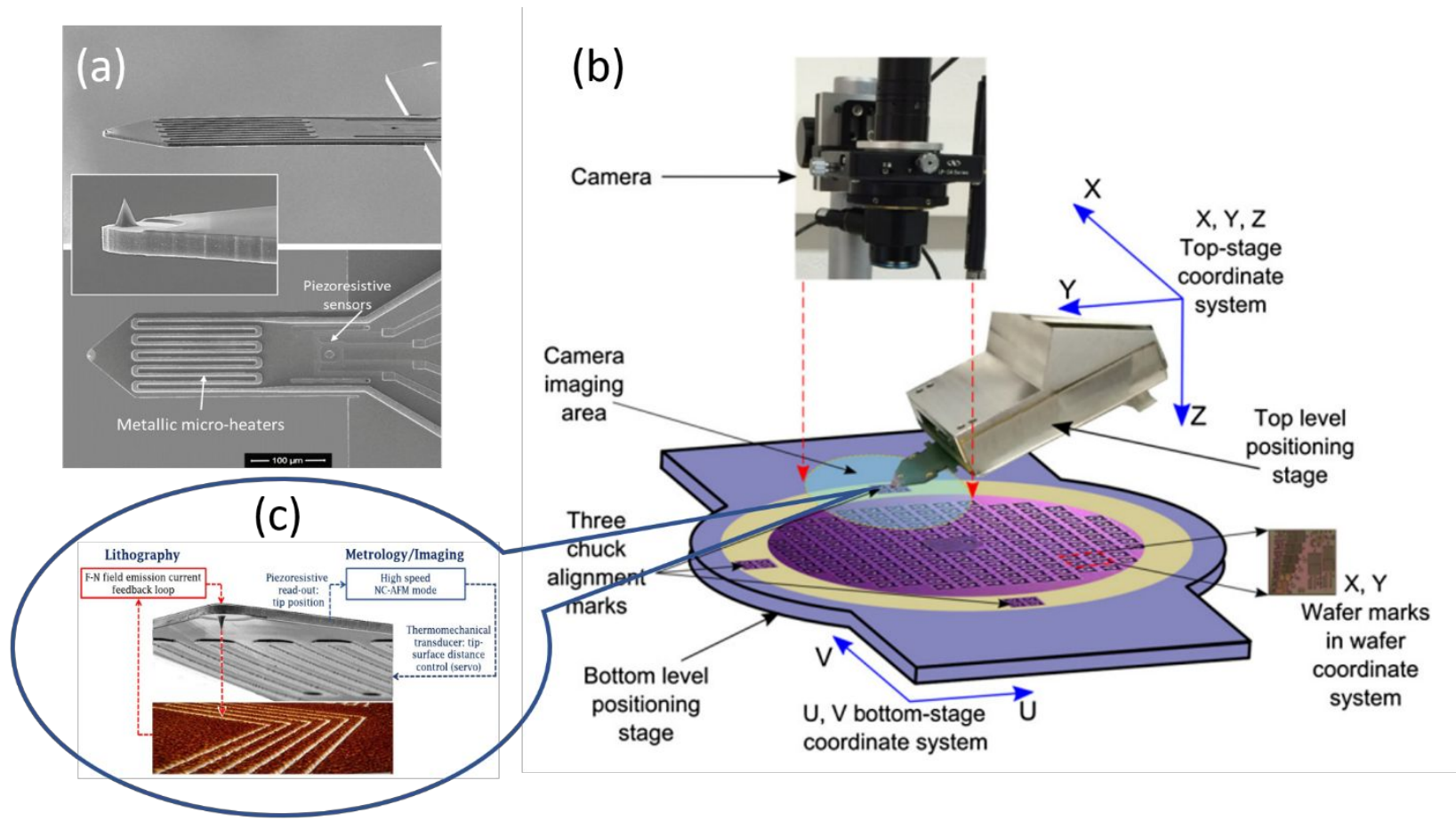

Figure S5. (a) SEM images of an actual active cantilever has been used in this research. Reprinted with permission from Ref. 2, (C [2019] IEEE, and Ref. 3, Copyright 2017, American Vacuum Society. (b) Schematic of an AFM setup equipped with corresponding sensors to incorporate active cantilever for nanoscale lithography and high-resolution imaging. Reprinted with permission from Ref. 1, Copyright 2015 SPIE. (c) Zoom-in schematic of an active cantilever in proximity of a sample for nanolithography. Reprinted with permission from Ref. 2, (C) [2019] IEEE. 
and its amplitude damping or frequency shift are used for tip-sample distance control and topography tracking. Therefore, microscopes incorporating them offer a better controllability and significantly better imaging throughput. Furthermore, in contrast to conventional optical read-out systems, these systems offer the potential for full lithographic and metrological automation. For Field Emission-Scanning Probe Lithography, the implemented active probes enable an in-situ inspection capability, a quantitative mapping at unprecedented resolution, as well as an integrated overlay alignment system. Figure S5 schematically demonstrate an active cantilever and its integrated sensors during lithography. ${ }^{4}$

\section{Different resist tones under FE-SPL nanopatterning}

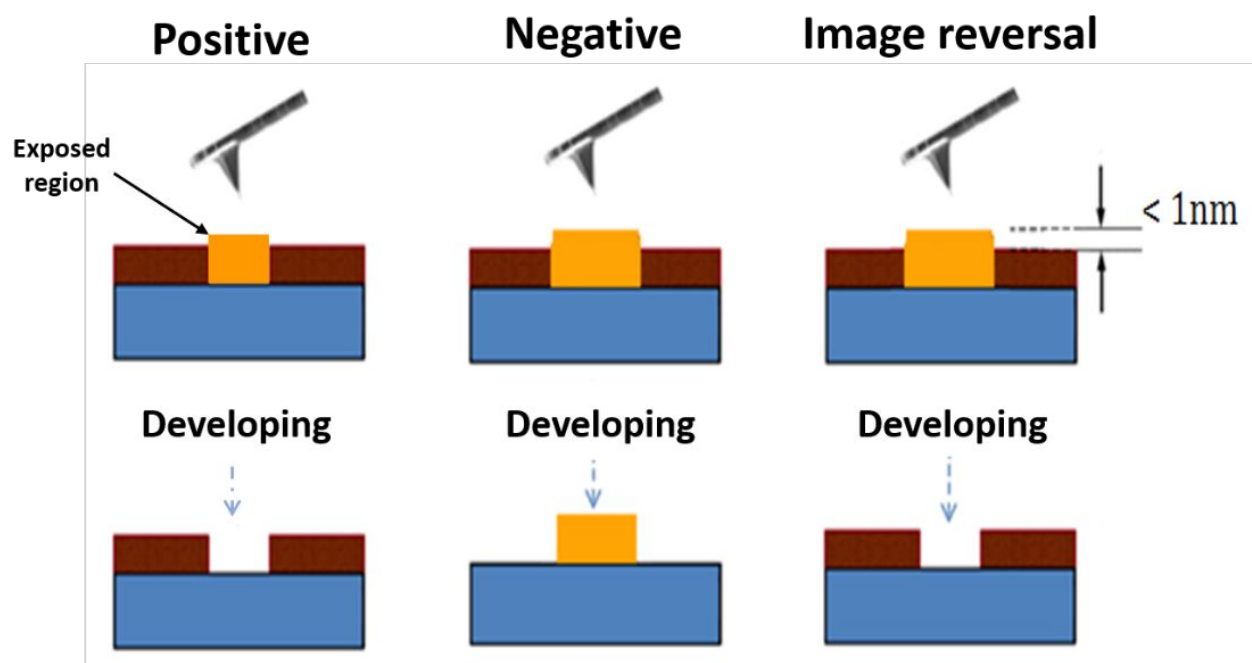

Figure S6. Basic lithographic tones in the FE-SPL. (a) positive (development less), (b) negative, and (c) image reversal.

\section{Si and GaN NW tips wear experiments}

Wear measurements of the tips were performed in contact mode on $<100>4$ "-Silicon wafer with root mean squared (RMS) roughness of $0.945 \mathrm{~nm}$ (manufacturer specification) and contact force load of $25 \mathrm{nN}$ at ambient condition. Scanning electron microscopy imaging of tip geometry were used to analyze the wear as shown in Figures S7 and S8. Stiffness of the active cantilevers was measured to be $56 \mathrm{~N} / \mathrm{m}(+/-2 \mathrm{~N} / \mathrm{m})$ using force displacement method. All sliding experiments were in fields with size of $100 \mu \mathrm{m} \times 100 \mu \mathrm{m}$ resulting in a total sliding distance of about $10 \mathrm{~mm}$ per field. Every tip has been slided 100 times on the same place without interrupting. The sliding velocity was $1 \mu \mathrm{m} / \mathrm{s}$. 

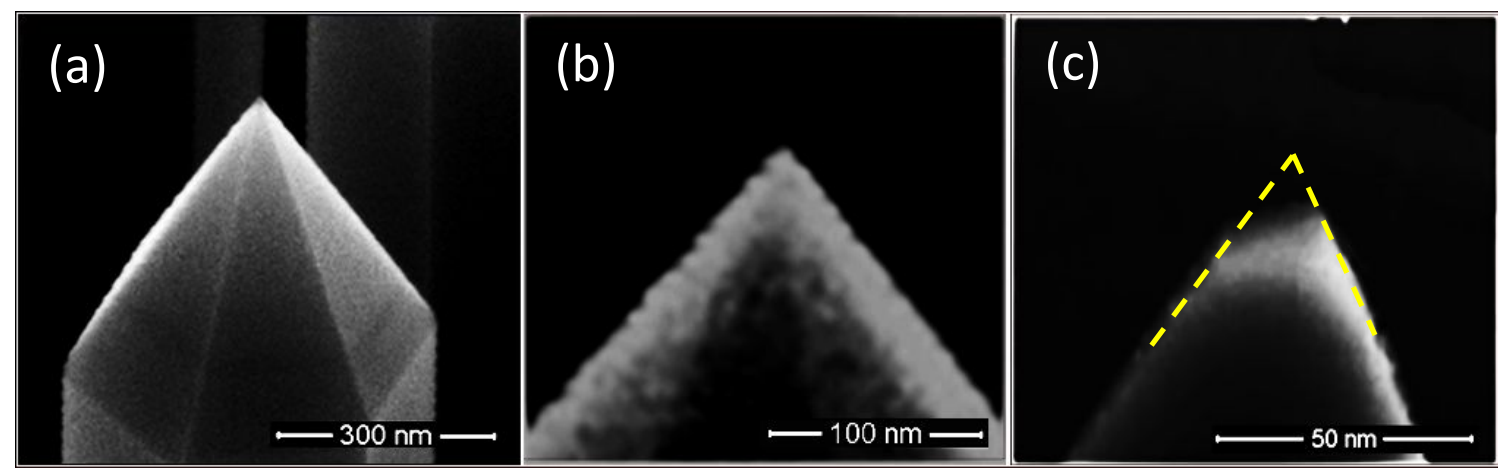

Figure S7: Scanning electron microscope images of a GaN tip (a) before, (b) after, and (c) wear tests.
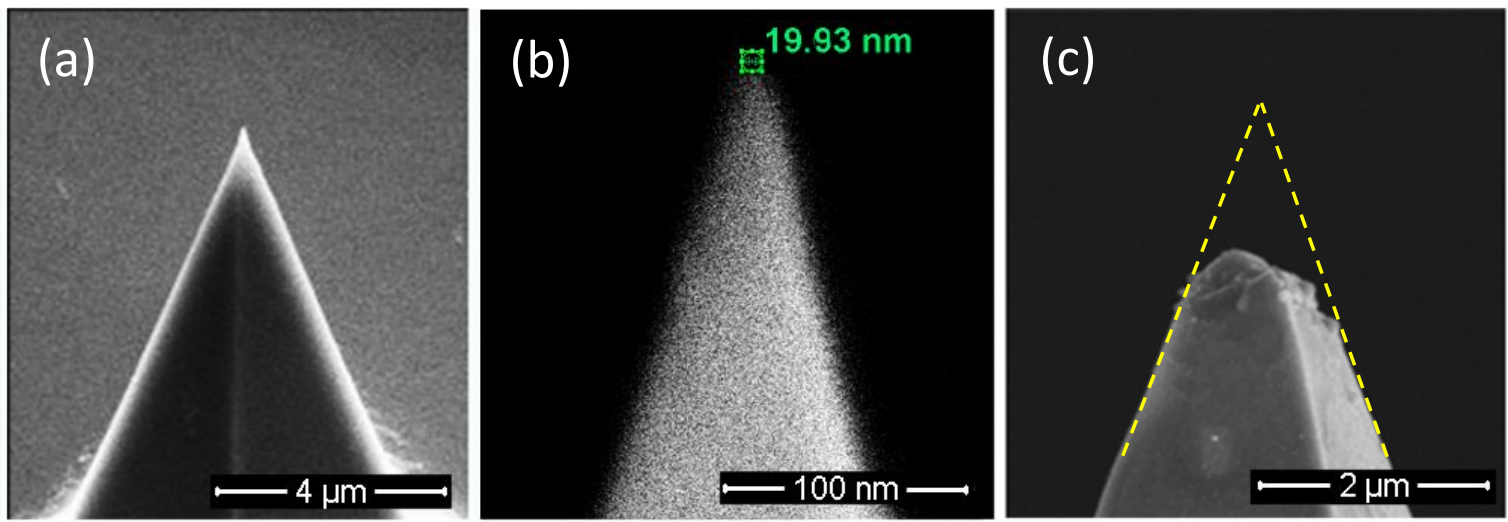

Figure S8: Scanning electron microscope images of a Si tip (a) before, (b) after, and (c) wear tests.

\section{References}

(1) Kaestner, M.; Aydogan, C.; Ivanov, T.; Ahmad, A.; Angelov, T.; Reum, A.; Ishchuk, V.; Krivoshapkina, Y.; Hofer, M.; Lenk, S. Advanced Electric-Field Scanning Probe Lithography on Molecular Resist Using Active Cantilever. Journal of Micro/Nanolithography, MEMS, and MOEMS 2015, 14 (3), 031202.

(2) Hofmann, M.; Mechold, S.; Holz, M.; Ahmad, A.; Ivanonv, T.; Rangelow, I. W. Active Cantilevers with Diamond-Tip for Field Emission Scanning Probe Lithography and Imaging. In 2019 IEEE International Conference on Mechatronics (ICM); IEEE, 2019; Vol. 1, pp 123-128.

(3) Rangelow, I. W.; Ivanov, T.; Ahmad, A.; Kaestner, M.; Lenk, C.; Bozchalooi, I. S.; Xia, F.; Youcef-Toumi, K.; Holz, M.; Reum, A. Active Scanning Probes: A Versatile Toolkit for Fast Imaging and Emerging Nanofabrication. Journal of Vacuum Science \& Technology B, Nanotechnology and Microelectronics: Materials, Processing, Measurement, and Phenomena 2017, 35 (6), 06G101.

(4) Active Cantilever Technology | Nano Analytik. 